\title{
TEORÍA DE LA VERDAD EVOLUCIONARIA EN PEIRCE
}

\author{
CRISTIAN SOTO \\ Departamento de Filosofía \\ Universidad de Chile \\ cssotto@gmail.com
}

RESUMEN: Expondré las líneas generales de una teoría de la verdad evolucionaria con tres objetivos principales: primero, mostrar que ella da cuenta de la teoría de la verdad pragmatista de Peirce, reuniendo la convergencia y la correspondencia; segundo, evidenciar que involucra compromisos metafísicos realistas a partir de la relación entre evolución, verdad y realidad; y, tercero, arrojar luz sobre un aspecto importante de la discusión contemporánea sobre teorías de la verdad, a saber, su carácter eminentemente metafísico.

PALABRAS CLAVE: evolución, realidad, convergencia, correspondencia, metafísica

SUMMARY: I shall expound the general lines of an evolutionary theory of truth with three main purposes: first, to show that it offers an account of Peirce's pragmatist theory of truth, taking together convergence and correspondence; second, to evidence that it involves realist metaphysical commitments in the relation between evolution, truth, and reality; and, third, to throw light on an important aspect of the contemporary discussion about theories of truth, namely, its eminently metaphysical character.

KEY WORDS: evolution, reality, convergence, correspondence, metaphysics

\section{Introducción}

Expondré las líneas generales de lo que llamaré teoría de la verdad evolucionaria en Peirce. Me interesará destacar sus compromisos metafísicos realistas, cuestión que resulta de primera importancia si se considera la discusión contemporánea sobre teorías de la verdad, en la que se han generalizado dos opiniones: primero, que una teoría de la verdad es metafísicamente neutral ( $c f r$., a propósito de Tarski 1944, Soames 1984, p. 414; en otros respectos Horwich 1996, p. 193; Resnik 1992, pp. 421 y ss.; Macintosh 1994, p. 153; y, a propósito de Peirce, Hookway 2002, pp. 50 y ss., y 2004, pp. 127 y ss.); y, segundo, que, en particular, la llamada teoría de la verdad pragmatista es instrumentalista, verificacionista $\mathrm{u}$ operacionalista (Quine 1953, pp. 42 y ss., especialmente la p. 46; Resnik 1992, pp. 422 y ss.; Ullian 1991, p. 64; Haack 1976, p. 232, aunque refiriéndose a Peirce, James y Dewey). ${ }^{l}$ En este trabajo discutiré directamente el

${ }^{1}$ Sólo para destacar la larga tradición interpretativa en relación con este punto, pueden tenerse en cuenta también los trabajos de George 1955; Hinshaw Jr. 1944; y Törnudd 1915. 
primer punto y ofreceré evidencia textual para rechazar el segundo y proponer, a partir de Peirce, una teoría de la verdad evolucionaria. ${ }^{2}$

De inmediato, también, conviene hacer la advertencia de que otros han intentado ofrecer un informe de la teoría de la verdad en Peirce. Tengo a la vista la propuesta antes mencionada de Hookway (2002, pp. 50 y ss., y 2004, pp. 127 y ss.), que problematiza la relación entre una teoría de la verdad y sus compromisos metafísicos; también la de Misak (1991), que enfatiza con claridad y, a mi entender, acertadamente en su capítulo 4 (1991, pp. 125 y ss.) la distinción entre verdad y realidad en Peirce. ${ }^{3}$ Asimismo, el trabajo de Forster resulta particularmente exhaustivo al evidenciar aspectos de la correspondencia, la coherencia, el instrumentalismo y el consensualismo en la propuesta peirceana ( $c f r$. Forster 1996, pp. 120 y ss.). En la misma dirección, Hynes (2006) examina textos correspondentistas y coherentistas, además de las lecturas de Rescher y Forster, para intentar una vez más mostrar la unidad de la propuesta de Peirce. ¿En qué se diferencia de los trabajos recién mencionados mi aproximación evolucionaria? Principalmente, en que si bien la cuestión de la unidad de la teoría de la verdad en Peirce es uno de los asuntos de mi investigación, no es en ningún caso el asunto principal, sino que éste más bien se focalizará en que la teoría de la verdad evolucionaria de Peirce es una teoría que evidentemente involucra compromisos metafísicos realistas.

\section{Teoría de la verdad evolucionaria}

¿Qué no es la teoría de la verdad evolucionaria? En principio, no es una teoría que sostenga que la verdad sea una propiedad de aquellas creencias que resultan biológicamente convenientes para la conservación de la especie o de un individuo. Hay pasajes en los que Peirce parece apuntar en aquella dirección, cuando sostiene que la creencia "es un estado de calma y satisfactorio que no deseamos abandonar, ni [tampoco queremos] cambiar la creencia por alguna otra, [sino que] nos adherimos tenazmente no sólo a la creencia, sino a creer justamente en lo que creemos" (EP 1, 1877, p. 115). Es el mismo Peirce, sin embargo, el que rechaza la tenacidad como método

${ }^{2}$ No pretendo que el análisis de la teoría de la verdad evolucionaria en Peirce cuente como la teoría de la verdad del pragmatismo. No siempre se ha hecho esta salvedad, pertinente en la medida en que difícilmente se puede elaborar un informe unificado de las propuestas de Peirce (EP 1, 1878), James (1907 y 1909) y Dewey (1911) acerca de la verdad.

3 "Verdad y falsedad son propiedades de las representaciones, pensamientos o proferencias, y de esta manera el asunto de la verdad puede ser indeterminado y la realidad subyacente determinada" (Misak 1991, p. xi; también pp. 125 y ss.) 
para fijar creencias ( $c f r . E P$ 1, 1877, pp. 116 y ss.), destacando con ello que no basta el mero impulso biológico para sostener la verdad de una creencia porque resulte adecuada para la vida, sino que ésta se acepta como verdadera solamente en la medida en que ha sido aprobada por el método de la fijación de creencias de la ciencia o de la experiencia, que no opera atendiendo a un criterio de adecuación biológica. La teoría de la verdad evolucionaria de Peirce se elabora dentro de su metafísica evolucionaria, basada en la relación estrecha entre evolución, verdad y realidad, y no dentro del evolucionismo científico darwiniano (cfr. Wiener 1946, pp. 223 y ss.; Fisch 1947, pp. 357 y ss.).

¿Qué es entonces la teoría de la verdad evolucionaria? En lo fundamental, es aquella que reúne las teorías de la convergencia y de la correspondencia. Por el lado de la convergencia sostiene que la verdad es el resultado de la evolución de los procesos epistémicos naturales del hombre y de los instrumentos disponibles para la investigación científica, que en su desarrollo se adaptan para el conocimiento del mundo. Y por el lado de la correspondencia sostiene que, más allá de los constreñimientos epistémicos propios de la investigación de la verdad, esta última tiene que entenderse como una creencia que se corresponde con algo real, involucrando en esto tanto una semántica realista para nuestras creencias como una ontología realista para el objeto referido por ellas. ${ }^{4}$

Con el propósito de desarrollar esta caracterización inicial de la teoría de la verdad evolucionaria, en lo que sigue argumentaré que se puede ofrecer un informe coherente y sistemático de las ideas de Peirce acerca de la convergencia y de la correspondencia como elementos de una única teoría de la verdad evolucionaria; mostraré, también, que la noción de verdad está en estrecha relación con la noción de realidad; y expondré, finalmente, que la concepción peirceana de la evolución involucra algunas determinaciones específicas sobre la naturaleza de la verdad y de su consecución, expresadas en el supuesto metafísico evolucionario de la adecuación entre la mente y el mundo, que encuentra un respaldo conceptual en las nociones de abducción y de innatismo naturalista ${ }^{5}$ desarrolladas por el filósofo.

\footnotetext{
${ }^{4}$ Más adelante, a propósito de la correspondencia, especifico lo que se entiende por semántica realista y por ontología realista como compromisos metafísicos de la teoría de la verdad evolucionaria. Me sirvo de la distinción de Psillos (2005, p. 385) para el realismo científico.

${ }^{5}$ En la sección inmediatamente siguiente, ilustro algunos elementos del innatismo naturalista peirceano en la segunda razón a favor de la complementariedad coherente y sistemática de la convergencia y la correspondencia. Lo llamo innatismo naturalista,
} 


\subsection{Convergencia y correspondencia}

$\mathrm{Al}$ examinar las ideas de Peirce acerca de la verdad, nos encontramos con dos tipos de aproximaciones, entre sí muy diferentes, que incluso podrían formularse como dos teorías de la verdad independientes: convergencia y correspondencia. Los comentaristas usualmente han identificado la teoría de la verdad de la convergencia como aquella que refleja la postura pragmatista, dejando sólo en un segundo plano la consideración de una teoría de la verdad de la correspondencia expuesta por el mismo Peirce ( $c f r$. Macintosh 1994, p. 153; Resnik 1992, p. 425; y Smart 1995, p. 313); y, acto seguido, dado que la convergencia involucra por sí misma constreñimientos epistémicos, se suele sostener que la teoría de la verdad pragmatista es inevitablemente antirrealista o, al menos, neutral respecto de la cuestión del realismo.

Esbozaré las líneas principales de la convergencia y de la correspondencia en Peirce, para mostrar que no sólo son coherentes entre sí, sino que incluso se complementan sistemáticamente dentro de una única teoría de la verdad pragmatista peirceana, que, dado el vínculo que establece con la realidad y con la evolución, conviene llamar teoría de la verdad evolucionaria.

El siguiente pasaje es ilustrativo respecto de la teoría de la verdad como convergencia, puesto que en él se destaca el proceso que se lleva a cabo a partir de ideas antagónicas que, en el progreso de la investigación, inevitablemente conducen a una misma conclusión:

Diferentes mentes pueden disponer de las convicciones más antagónicas, pero el progreso de la investigación las conducirá por fuerza fuera de ellas mismas hacia una y la misma conclusión. Esta actividad del pensamiento por la cual somos conducidos, no a lo que deseamos, sino a un objetivo previamente ordenado [a preordained goal], es como la operación del destino. (EP 1, 1878, p. 138)

Es destacable en este pasaje la relación que se establece entre, primero, la situación particular del sujeto epistémico que investiga; segundo, el progreso en la investigación, que en cada caso está sometido a las variables propias de la economía de la investigación de una comunidad científica (tiempo, esfuerzo, dinero, etc.); y, tercero, la conclusión, al parecer metafísicamente insoslayable, a la que se tiene

aunque Peirce no emplee esos términos, porque se refiere al estatus de ciertas creencias básicas innatas, en el sentido de naturales, que son el resultado de la evolución adaptativa de la mente al mundo. 
que llegar a través de la investigación, que es, según Peirce, como la operación del destino. Hay, en este pasaje, un punto de primera importancia: incluso en la teoría de la verdad de la convergencia se alude tanto a los procesos epistémicos como a ciertos elementos metafísicos, ambos complementarios entre sí a la hora de describir la investigación científica. El pasaje, sin embargo, continúa enfatizando los supuestos metafísicos al señalar que el proceso encarna una gran ley que conduce a la opinión predestinada, que expresa una creencia cuyo correlato es lo real:

Ninguna modificación del punto de vista que se asuma, ninguna selección de otros hechos para el estudio, ni siquiera una inclinación natural de la mente, puede hacer capaz a un hombre de escapar a la opinión predestinada [predestinate opinion]. Esta gran ley está encarnada en la concepción de la verdad y de la realidad. La opinión que está destinada [is fated] a ser finalmente acordada [agreed] por todos los que investigan es lo que entiendo por verdad, y el objeto representado por esta opinión es lo real. Ésta es la manera en que yo explicaría la realidad. (EP 1, 1878, p. 139)

La teoría de la verdad de la convergencia da cuenta de una aproximación metafísica a la realidad y de una aproximación que, precisamente, es compatible con las condiciones epistémicas específicas del agente y con los instrumentos disponibles para la investigación científica en un momento dado. El supuesto metafísico de fondo es, en este pasaje, que, no obstante los constreñimientos epistémicos, hay una opinión predestinada que expresa una creencia verdadera, cuyo objeto es lo real. El mismo Peirce subraya que su teoría de la verdad de la convergencia es una manera de explicar la realidad.

El pasaje recién analizado data de 1878. Otro pasaje de 1911 confirma la misma idea y muestra la continuidad de los planteamientos de Peirce. Allí se sostiene: "Llamo verdad a la opinión predestinada, por la cual entiendo aquella que prevalecería finalmente si la investigación fuera conducida suficientemente lejos" (EP 2, p. 457). Esto respalda la tendencia de los comentaristas a identificar la teoría de la verdad de la convergencia con la teoría de la verdad del pragmatismo peirceano. Sin embargo, tal tendencia tiene que ser matizada poniendo de relieve dos puntos: primero, que esta teoría de la verdad de la convergencia involucra no sólo consideraciones epistémicas, sino también metafísicas, que apuntan a la relación entre verdad, realidad 
y evolución; y, segundo, que hay también una teoría de la verdad de la correspondencia. Paso ahora a este segundo punto. ${ }^{6}$

Peirce define su teoría de la verdad de la correspondencia sosteniendo que "la verdad consiste en un hecho real que se corresponde con una proposición verdadera" (EP 1, 1878, p. 147). Sus implicaciones metafísicas son evidentes: una proposición verdadera expresa una creencia que asegura, por su naturaleza, una referencia a la realidad. Es a este respecto que la teoría de la verdad de la correspondencia resulta más adecuada para un realismo semántico, basado en las propiedades intencionales de las creencias que son referenciales, y para un realismo ontológico, basado en las propiedades de aquello a lo que se refiere la creencia. Es a esto a lo que llamaré en lo sucesivo "compromisos metafísicos de la teoría de la verdad evolucionaria".

Las razones para apoyar la complementariedad coherente y sistemática de la convergencia y de la correspondencia resultan evidentes. La primera es ésta: se tiene que reparar en que de los pasajes analizados se desprende que la convergencia y la correspondencia son compatibles en la medida en que la primera conduce a la segunda, es decir, el estado ideal postulado por la convergencia de los investigadores en una opinión predestinada, que es la verdadera, no es sino un estado de creencia que se corresponde con el estado de cosas del mundo. Ahora bien, ¿qué se quiere decir con predestinación en la formulación de la convergencia? No otra cosa que una predestinación natural, esto es, una opinión cuya verdad depende de la correspondencia con lo real, a la que se tiene que llegar inevitablemente si la investigación de la naturaleza es conducida suficientemente lejos.

La segunda razón apela al innatismo naturalista y a que debe tenerse a la vista la adecuación entre la mente y el mundo desarrollada por Peirce y que cuenta como el supuesto metafísico evolucionario fundamental, inspirado en el evolucionismo científico de Darwin. A partir de tal supuesto se entiende que la convergencia hace referencia al proceso evolutivo natural en el que las capacidades epistémicas se han adaptado para el conocimiento del mundo. Peirce sostiene que "parece incontestable, por consiguiente, que la mente del hombre está fuertemente adaptada para la comprensión del mundo" (EP 1, 1878, p. 181). Señal de esto es que, "al menos, hasta donde esto corre,

\footnotetext{
${ }^{6}$ Tal vez se vea cierto contrasentido en seguir distinguiendo entre convergencia y correspondencia, cuando lo que se quiere mostrar es la unidad de las mismas en una única teoría de la verdad evolucionaria. Por cierto, es este último el propósito principal. Sólo atendiendo a las distinciones habituales en la literatura al respecto caracterizo separadamente la convergencia y la correspondencia.
} 
hay ciertas concepciones, sumamente importantes para tal comprensión, que surgen con naturalidad en su mente; y sin tal tendencia la mente jamás puede llegar a tener ningún desarrollo en absoluto" (EP 1, 1878, p. 181). Estas concepciones forman parte del innatismo naturalista peirceano, que el autor caracteriza sosteniendo: "¿cómo tenemos que explicar esta adaptación [de la mente al mundo]? Las grandes utilidades y la indispensabilidad de la concepción del tiempo, del espacio y de la forma, incluso en las más bajas inteligencias, son tales como para afirmar que ellas son el resultado de la selección natural" (EP 1, 1878, p. 181). Es así, entonces, como creencias del tipo tiempo, espacio y forma constituyen parte del repertorio conceptual del innatismo naturalista, que es la hipótesis que confirma la adecuación de la mente para el conocimiento del mundo. La relación del innatismo naturalista con la convergencia salta a la vista, puesto que aquellas concepciones sumamente importantes para la investigación científica y para el conocimiento del mundo surgen de manera natural en la mente del hombre como resultado de la evolución por selección natural. Aun cuando la hipótesis del innatismo naturalista pueda contar como una hipótesis empírica, es clara su vinculación con el supuesto fundamental de la metafísica evolucionaria, que afirma que la naturaleza de la mente se adapta por sus necesidades al conocimiento del mundo. Así, al menos tres momentos se distinguen en la convergencia: primero, el proceso de investigación, epistémicamente constreñido; segundo, el consenso final de los miembros de la comunidad científica; y tercero, la correspondencia mente-mundo propiamente tal, cuya dirección de ajuste va de la naturaleza del mundo a la naturaleza de lo mental. ${ }^{7}$

De todos modos, se reconoce que la convergencia, más que la correspondencia, involucra factores epistémicos que constriñen la consecución de la verdad. Entre esos factores, además de las capacidades epistémicas del agente, se encuentran el método de investigación empleado y la teoría científica disponible, que en el momento resultan más adecuados para un caso específico. El problema que se enfrenta surge al considerar que, si bien la convergencia conduce al descubrimiento de lo que, en términos de la metafísica evolucionaria, es la opinión predestinada, no quedan claros cuáles sean los criterios que permiten garantizar que la opinión en la que, finalmente, resultan

${ }^{7}$ Por cierto, en Peirce también se plantea el problema de la relación mentemundo, si acaso es causal, si acaso forma parte de una única naturaleza, etc. Sobre todo a partir de la noción de abducción pueden extraerse algunas luces a este respecto. Por ahora sólo me interesa advertir que no paso por alto los problemas que arrastra el supuesto del ajuste mente-mundo del que hago uso en mi argumento. 
converger los investigadores es efectivamente la opinión verdadera. El mismo Peirce se refiere a este punto al señalar que la distinción entre las creencias verdaderas y las falsas "pertenece, en su desarrollo completo, exclusivamente al método científico para fijar la opinión" (EP 1, 1878, p. 137); es, pues, el método científico el que tiene que poner los criterios para distinguir entre las creencias pretendidamente verdaderas y las que efectivamente lo son; y tal vez atendiendo a este punto a la metafísica evolucionaria de Peirce se la llama también "metafísica científica", al menos en el sentido de que sustenta un realismo científico ( $c f r$. Psillos 2005, pp. 385 y ss; Sankey 2008, pp. 12 y ss; y Soto 2009, pp. 23 y ss).

\subsection{Verdad y realidad}

Un primer paso que deja a la vista los posibles compromisos metafísicos realistas de una teoría de la verdad evolucionaria consiste en sostener que las creencias verdaderas son creencias que tienen por correlato algo real, mientras que las creencias falsas son creencias en meras ficciones ( $c f r$. EP 1, 1878, p. 37). La teoría de la verdad evolucionaria vincula estrechamente verdad y realidad. A propósito de la convergencia he citado el pasaje en el que Peirce afirma que "la opinión que está destinada a ser acordada finalmente por todos los que investigan, es lo que entiendo por verdad, y el objeto representado en esta opinión es lo real" (CP 5.417, y $W 3$, p. 273). Y, asimismo, a propósito de la correspondencia he citado el pasaje en el que afirma que "la verdad consiste en un hecho real que se corresponde con una proposición verdadera $(E P$ 1, 1878, p. 147). En ambas formulaciones la verdad está vinculada con la realidad.

Un segundo paso consiste en analizar qué se entiende por realidad, cuestión que se aborda desde el pragmatismo peirceano de diversas maneras, todas coincidentes en una fuerte impronta realista. Una primera definición, sumamente ilustrativa, señala que "lo real es aquello cuyas características son independientes de lo que cualquiera pueda pensar acerca de ello" ( $W$ 3, p. 271). La independencia respecto del ámbito de lo mental se enfatiza en las siguientes líneas:

[O]bsérvese que "lo externo" significa simplemente aquello que es independiente de qué fenómeno esté inmediatamente presente, esto es, de cómo podamos pensar o sentir; del mismo modo que "lo real" significa aquello que es independiente de cómo podamos pensar o sentir acerca de ello [...]. Queda claro que esta concepción de la realidad es inevitablemente realista. $(E P 1,1871$, p. 90) 
Conviene reparar de inmediato en que no se identifica lo externo con lo real; simplemente se establece una semejanza, puesto que efectivamente para Peirce lo real no se limita a los objetos físicos externos, tal como usualmente se entiende esta expresión. ${ }^{8}$ Lo relevante por ahora está en atender a que lo real es aquello que es independiente de cómo nosotros podamos pensarlo o sentirlo y, en este dirección, es ontológicamente independiente de las constricciones epistémicas que en un momento particular determinen el éxito de la investigación científica. Con lo dicho queda a la vista que el hecho de que lo real sea ontológicamente independiente de nuestros procesos epistémicos, es una suposición necesaria para poder sostener que hay una opinión predestinada, que expresa una proposición verdadera, que se corresponde con lo real; la verdad en la teoría de la verdad evolucionaria no depende en ningún caso de lo que los investigadores quieran sentir o pensar, sino sólo del resultado natural del proceso de investigación, si se lleva suficientemente lejos.

\subsection{Evolución y verdad}

La influencia del evolucionismo en Peirce y, en general, en la filosofía americana ha sido abordada brillantemente por Wiener (1946, pp. 321 y ss.) y Fisch (1947, pp. 357 y ss.). En particular, el carácter evolucionario de la teoría de la verdad que propongo puede ilustrarse desarrollando las nociones de abducción y descubrimiento, puesto que a propósito de ellas se implementa el supuesto metafísico evolucionario de la adecuación de la mente para el conocimiento del mundo.

La teoría de la verdad evolucionaria establece una relación específica entre abducción y verdad al sostener que la verdad "sólo puede provenir de la abducción" (EP 2, 1901, p. 107); a diferencia, entonces, de la deducción y de la inducción, la abducción es la única especie de inferencia que descubre nuevas verdades. $\mathrm{Y}$ dado que la verdad es el tipo de creencia que se corresponde con lo real, cabe preguntarse: ¿se ponen en juego en el descubrimiento abductivo tanto la verdad como la realidad? En el siguiente pasaje se describe la abducción a partir de la afinidad entre la mente y la verdad, que descansa en la existencia de un instinto natural de la primera hacia

\footnotetext{
${ }^{8}$ No tiene caso desarrollar esta distinción metodológica aquí, aunque tenga fuertes consecuencias para la ontología, es decir, para la concepción que se tenga de lo real. Por lo pronto, conviene tener a la vista el trabajo de Peirce (EP 2, 1911, pp. 434-450) titulado A Neglected Argument for the Reality of God, en el que se defiende la realidad de summum ens sirviéndose de la descripción de la investigación científica basada en los tipos de inferencias.
} 
la segunda: "Es una hipótesis primaria que subyace a toda abducción que la mente humana es afín a la verdad en el sentido de que en un número finito de conjeturas arrojará luz sobre la hipótesis correcta. [... L La existencia de un instinto natural hacia la verdad es, después de todo, la gran ancla de la ciencia" (EP 2, 1901, p. 108).

La hipótesis primaria a la que se hace referencia aquí tiene que considerarse como una abducción fundamental, una reformulación del llamado supuesto fundamental de la metafísica evolucionaria, sobre la que se asientan las abducciones particulares de la ciencia. Se destaca en estas líneas el instinto natural hacia la verdad, que, por cierto, comporta las características de una hipótesis empírica, la cual lo hace semejante a los instintos de las hormigas y de las abejas, pero también las características de una hipótesis metafísica, en la que se asume que la mente, gracias a su evolución adaptativa natural, es afín a la verdad. Es éste, tal vez, el sustrato más profundo de la teoría de la verdad evolucionaria, puesto que, desde sus planteamientos, no podría explicarse el descubrimiento de verdad alguna sin suponer esta abducción fundamental, que es la hipótesis de que la mente se ha adecuado natural y evolutivamente para el descubrimiento de la verdad, el conocimiento de la realidad.

A propósito de la relación entre metafísica evolucionaria y verdad, un último punto tiene que ver con los compromisos metafísicos que arrastra la abducción para la ontología. Si la verdad es aquella creencia que se corresponde con lo real, entonces la abducción es aquel proceso epistémico natural que nos pone en relación con la realidad y con su conocimiento en el descubrimiento científico; es, en otros términos, nuestro acceso epistémico, evolutivamente fundado, a la realidad.

\section{Compromisos metafísicos realistas}

La lectura que propongo de una teoría de la verdad evolucionaria, que involucra compromisos metafísicos realistas, ha encontrado oposición en los trabajos de Hookway (2002, pp. 50 y ss., y 2004, pp. 127 y ss.), que defienden que una teoría de la verdad pragmatista peirceana es neutral respecto de cualquier compromiso metafísico. En relación con esto, el trabajo de Hookway comparte una postura generalizada acerca de la neutralidad de una teoría de la verdad respecto de las cuestiones metafísicas que tienen que ver con la semántica y la ontología ( $c f r$. Soames 1984 y Horwich 1996, antes citados). Para sostener mi propuesta, inspirada en la línea de Smart ( $c f r$. principalmente 1995, p. 301, y 1963, pp. 651 y ss.) y Vallejos (1996, pp. 2, 22 y ss.), 
revisaré las objeciones de Hookway con el objetivo de ofrecer una estrategia de lectura que distinga las consideraciones epistémicas y metafísicas en una teoría de la verdad y que permita mantener en pie los compromisos metafísicos realistas de la propuesta evolucionaria.

\subsection{Hookway y la neutralidad metafísica de la teoría de la verdad en Peirce ${ }^{9}$}

Hookway afirma que el "informe de la verdad [de Peirce] es metafísicamente neutral. Puede sostenerse en conjunción con una metafísica realista o con una antirrealista" (2002, p. 77). Su argumento central en apoyo de esta afirmación se basa en distintos casos que parecen romper el vínculo entre verdad y realidad. Según Hookway, los textos de Peirce exigen aceptar que para algunas entidades y disciplinas es aplicable una teoría de la verdad con compromisos metafísicos realistas, esto es, que la verdad se formule en términos de una proposición que es verdadera respecto de algo real, mientras que para otras entidades y disciplinas se requiere una teoría de la verdad que no tenga tales compromisos.

La concepción más plausible sería que algunas verdades pueden ser entendidas de una manera "realista", en cuanto que tienen que ver con la realidad independiente de la mente, mientras que otras tienen que ver con asuntos cuyo carácter ostenta características [marks] de nuestros intereses, sentimientos o actividades constructivas. (Hookway 2002, p. 77)

Su argumentación sigue dos líneas; en primer lugar describe casos en los cuales, a pesar de haber algo real, no hay verdades; y, en segundo, describe casos en los cuales, a pesar de haber verdades, no hay algo que pueda considerarse real, en el sentido de independiente de la mente y de la percepción humanas.

Entre los casos en los que hay algo real, pero, aun así, no hay verdad, se encuentran las cualidades primarias y secundarias y los hechos perdidos u ocultos. Respecto del primero se sostiene que "nosotros esperamos que las cualidades primarias se muestren en leyes con un amplio espectro de aplicación explicativa y con la habilidad de unificar gran parte de nuestro conocimiento" (2002, p. 92), mientras que

${ }^{9}$ No es casual que sometamos a análisis la lectura de Hookway. También desde el punto de vista de Misak (1991, p. vii), la contribución de Hookway respecto del tema de la verdad en Peirce es una de las más significativas, junto a la de David Wiggins (2004). 
"las cualidades secundarias involucran similaridades que son relativas a nuestro aparato sensorial, como nuestra manera de responder a los colores" (2002, p. 92). La distinción que cobra relevancia aquí se asienta en las ideas de Locke ( $c f r$. 1979, libro II, capítulo VII, $\S \S 7-$ 26), quien sostiene que las cualidades primarias señalan cualidades reales de las cosas, tales como el peso, la forma, etc., mientras que las secundarias sólo indican la relación con nuestro modo de conocer tales cosas, por ejemplo, nuestro aparato visual para los colores o nuestros oídos para los sonidos. Hookway reconoce dos alternativas: por un lado, que puede considerarse que tanto las cualidades primarias como las secundarias son reales, caso en el que nos encontraríamos ante un realismo; y, por otro, que sólo las primarias son reales, caso en cual nos encontraríamos ante un nominalismo. Pero, concluye que, sea como fuere, la teoría de la verdad de Peirce es metafísicamente neutral a este respecto y no puede ayudar a dirimir el asunto ( $c f r$. Hookway 2002, pp. 93-94). Sin embargo, él mismo repara en que "la concepción realista de Peirce acerca de la realidad parece exigirle que tome la primera concepción" (2002, p. 94) y hace referencia al siguiente pasaje:

Los metafísicos que se dedican a la ontología usualmente dicen que las "sensaciones secundarias", tales como los colores, son ilusorias y falsas; pero no así el Pragmaticista. Él insiste en que la rosa realmente es roja; rojo es, por el significado de la palabra, una apariencia; y decir que la rosa Jaqueminot es roja significa, y puede significar, nada más que si tal rosa es puesta ante una vista normal, a la luz del día, se verá roja [it will look red]. (CP 8.194; cfr. Hookway 2004, p. 136)

Me referiré muy brevemente a los casos en los que hay verdades, pero parece no haber hechos. En este aspecto son interesantes los casos de la moral y de la matemática. El moralista que describe el ideal del summum bonum puede trazar el desarrollo de la naturaleza moral del hombre que lo conduciría a conformarse a tal ideal. Y puede creerse en la verdad del summum bonum así descrito. Sin embargo, tal ideal puede no corresponderse con realidad alguna que satisfaga sus descripciones. Vale decir, aun cuando pueda aceptarse la verdad del ideal del summum bonum dentro de una teoría moral, ella podría no corresponderse con nada en la realidad ( $c f r$. Hookway 2002, p. 76), por lo cual cabría aceptar que hay una creencia sin un correlato real en la acepción peirceana. En cuanto a las verdades matemáticas, el asunto parece más evidente, puesto que ellas son dependientes del pensamiento, contradiciendo el supuesto realista de 
la independencia de la realidad respecto del pensamiento humano. Es decir, si la realidad, que es el objeto de la verdad, es aquello que es independiente de cómo lo pensemos o lo percibamos, las entidades matemáticas parecen no cumplir con el criterio. Sin embargo, el siguiente pasaje rescatado por Misak (2004b, p. 167) resulta ilustrador: "Un metafísico podría sostener que esta misma fuerza que hace que los matemáticos acepten las proposiciones para las cuales ellos no estaban preparados, prueba e incluso constituye el modo de ser independiente del pensamiento matemático y, de esta manera, es una realidad" (CP 5.567, 1901).

La intención de Hookway consiste en mostrar que los casos mencionados rompen la relación estrecha entre verdad y realidad, que está a la base de una interpretación realista de la teoría de la verdad de Peirce y que, por ende, rota esta relación, se estaría en condiciones de sostener su neutralidad metafísica. No obstante, cabe insistir en que desde la aproximación evolucionaria puede ofrecerse una estrategia de lectura para evidenciar que la teoría de la verdad en cuestión ostenta compromisos metafísicos realistas.

\subsection{Consideraciones epistémicas y metafísicas de una teoría de la verdad}

Para evaluar los argumentos de Hookway y para entender de mejor manera el alcance de la teoría de la verdad evolucionaria, es preciso distinguir entre consideraciones epistémicas y metafísicas. Las metafísicas tienen por objeto dirimir qué es la realidad y qué es la verdad; mientras que las epistémicas tienen por objeto indagar cuál es el acceso que tiene el investigador a dicha verdad y a dicha realidad. Distinguir entre aspectos metafísicos y epistémicos no es, por cierto, algo nuevo en la discusión contemporánea; hay importantes trabajos de Smart (1995, pp. 302-303 y ss.; cfr. también 1963, 1982 y 1986) en los que explicita esta diferencia atribuyéndole especial importancia para la cuestión de la naturaleza de la verdad; y en la misma línea, pero centrado principalmente en la semiótica de Peirce, Vallejos (1996, pp. 1-2 y 22) destaca la impronta realista escolástica y metafísica de la noción de verdad. El trabajo sistemático, de todos modos, todavía no ha sido hecho y en la literatura sobre el tema parece predominar la opinión de que una teoría de la verdad es metafísicamente neutral: ${ }^{10} \sin$ desconocer los frutos de esta línea de

${ }^{10}$ Es difícil precisar si esta lectura proviene de la influyente concepción semántica de la verdad de Tarski (1944), como si fuera este trabajo el que ha dado lugar a que la noción de verdad se examine en la única dirección orientada al análisis de la 
investigación, tiene que reconocerse que, habiendo sido predominante, ha ocasionado que se desatiendan los aspectos metafísicos de la discusión contemporánea en torno a las teorías de la verdad.

Teniendo a la vista la distinción entre consideraciones metafísicas y epistémicas, puede ponerse de relieve que los casos de Hookway que intentan romper el vínculo realista entre verdad y realidad no son suficientes para sostener la neutralidad metafísica de la teoría de la verdad evolucionaria, puesto que ellos sólo tienen que ver con consideraciones epistémicas. El caso de las cualidades primarias y secundarias examina el acceso epistémico que el investigador tiene a los colores de la rosa, siguiendo el ejemplo mencionado; asimismo, el caso de los hechos perdidos u ocultos pone en tela de juicio el acceso epistémico que el historiador tiene a un evento determinado del pasado; el caso de las verdades morales pone en duda el acceso epistémico que tiene el moralista, dadas las condiciones y circunstancias de una época, a presenciar la realidad que corresponde a su idea del summum bonum; y, en el mismo sentido, el caso de las verdades matemáticas enfatiza el acceso epistémico que un investigador tiene al trabajo en matemáticas, en lugar de la naturaleza de las entidades matemáticas, que, conforme a Peirce, siguen su propia necesidad. Ninguno de estos casos tiene que ver directamente con las cuestiones de qué sea la verdad y qué sea la realidad, cuestiones que, por el contrario, pertenecen a las consideraciones metafísicas. No puede sostenerse que la teoría de la verdad evolucionaria sea metafísicamente neutral a partir de argumentos que sólo tocan cuestiones epistémicas. Lo que es más, en el siguiente pasaje queda a la vista que de algún modo el mismo Peirce tenía presente la distinción entre consideraciones epistémicas y metafísicas:

Todo pensamiento y opinión humanos contienen elementos arbitrarios, accidentales, dependientes de las limitaciones de las circunstancias, del poder y de las tendencias de lo particular; un elemento de error, en resumen. Pero la opinión humana tiende a la larga universalmente a una forma definida, que es la verdad. Permítase a cualquier ser humano tener suficiente información y ejercer suficientemente el pensamiento sobre alguna cuestión y el resultado será que llegará a una cierta conclusión definida, que es la misma que alcanzará cualquier otra mente bajo circunstancias suficientemente favorables. (EP 1, 1871, p. 89)

verdad de las proposiciones en lenguas artificiales o metalenguajes. Otro caso difícil de situar en la discusión es el de Kripke (1975), que por su trabajo en metafísica requiere, a mi entender, un análisis distinto. 
Epistémicamente, es cierto que todo pensamiento y opinión humanos están sujetos a un elemento de error, impuesto por los constreñimientos epistémicos del agente y por las limitaciones que impongan los instrumentos y las teorías científicas disponibles. Pero metafísicamente puede sostenerse que la opinión humana tiende, a la larga, a una forma definida, que es la verdad y que cualquier mente puede alcanzar bajo condiciones favorables en la investigación. Peirce adopta este último punto de vista, ligado a las consideraciones metafísicas, al referirse a la adecuación de la mente al mundo y a la generación natural de ciertas concepciones cuando afirma, por ejemplo, que "parece incontestable, por consiguiente, que la mente del hombre está fuertemente adaptada para la comprensión del mundo" (EP 1, 1878, p. 181).

Aún más, el mismo Peirce se refiere a una naturaleza de la verdad que es transversal a las verdades particulares de las ciencias:

Ahora, las diferentes ciencias tienen que ver con diferentes especies de verdad; la verdad matemática es una cosa, la verdad ética es otra, y la del estado del universo existente efectivamente [actually] es una tercera; pero todas aquellas concepciones tienen en común algo muy característico y claro. Todos nosotros esperamos que las diferentes investigaciones científicas en las cuales de diversa manera estamos involucrados tiendan finalmente hacia una conclusión definidamente establecida, conclusión a la cual intentamos anticiparnos en alguna medida. El acuerdo con esta proposición última que buscamos anticipar — el acuerdo con esto, sea el que fuere, es la verdad científica-. (CP 7.187, 1901; cfr. Misak 2004b, pp. 166-167)

Se destaca que todas las ciencias tienden por igual a la verdad y de la siguiente manera: descubriéndola. El trabajo del hombre de ciencia consiste en descubrir una verdad que es, en su naturaleza, independiente de él. Se trata de un descubrimiento que es posible gracias a la investigación científica y de manera diferente en cada disciplina, atendiendo a su metodología y a su objeto.

Éstas son las consecuencias que se obtienen al distinguir entre consideraciones epistémicas y metafísicas en una teoría de la verdad evolucionaria. Por una parte, es evidente que para que se dé la convergencia $y$, por ende, la correspondencia, hay algunas condiciones que dependen de las circunstancias en que se encuentra el investigador; y es cierto que ello, a primera vista, parece ser contrario a la idea realista de que la verdad tiene por objeto una realidad que es 
independiente de cómo la pensemos o la percibamos, porque, epistémicamente, el individuo no puede pensar o percibir sino bajo sus condiciones. Pero, por otra parte, también es evidente que la realidad y la verdad involucradas por la teoría de la verdad evolucionaria descansan en concepciones metafísicas, como la adecuación de la mente al mundo, el innatismo naturalista y la abducción, entre otros, que son los elementos teóricos que sustentan los compromisos metafísicos realistas.

\section{4. ¿Por qué una teoría de la verdad evolucionaria?}

El análisis de la teoría de la verdad evolucionaria peirceana nos permite obtener algunos resultados tanto históricos e interpretativos como sistemáticos.

Desde un punto de vista histórico, uno de los resultados relevantes consiste en evidenciar que los distintos intentos de esbozar una teoría de la verdad pragmatista (Horwich 1996; Soames 1984; Haack 1976; también George 1955; Hinshaw 1944; Törnudd 1915) ofrecen sólo caracterizaciones parciales de la misma, que en ningún caso dan cuenta de una única teoría de la verdad del pragmatismo. Del mismo modo, quienes han intentado ilustrar la unidad de la teoría de la verdad peirceana (Misak 1991; Forster 1996; y Hynes 2006), por ser distintos sus propósitos, no han enfatizado suficientemente sus compromisos metafísicos realistas. La teoría de la verdad evolucionaria es el esbozo que más se ajusta a la concepción de la verdad desarrollada por Peirce desde una perspectiva metafísico-evolucionaria dentro de su pragmatismo.

Desde una perspectiva sistemática, en cambio, las consecuencias que se obtienen nos permiten justificar la posición de la teoría de la verdad evolucionaria dentro de la discusión contemporánea. El mismo Soames (1984, p. 411) reconoce que puede desarrollarse una teoría de la verdad con propósitos filosóficos más amplios. En el presente caso, cabe entender esto como el vínculo estricto que se evidencia entre la teoría de la verdad evolucionaria y sus compromisos metafísicos realistas. Desde esta perspectiva destacaré cuatro resultados sistemáticos.

El primer resultado se desprende de la relación entre verdad y realidad, que tiene en su base la noción de evolución. La verdad, tanto en la convergencia como en la correspondencia, es la propiedad que poseen aquellas creencias que se refieren a algo real (sin que lo real, por cierto, quiera decir sólo objetos externos; cfr. la nota 8); y el origen del conocimiento, junto a cada uno de los descubrimientos de 
la ciencia moderna, se explica en este caso apelando a la evolución, que en términos de la metafísica evolucionaria peirceana consiste en la paulatina adecuación de los procesos epistémicos (mente, instrumentos y teorías disponibles) para el conocimiento del mundo, enfatizando en este punto principalmente la adecuación de la mente al mundo como elementos de un único orden natural.

El segundo resultado tiene que ver con la naturaleza de la verdad que sustenta la teoría de la verdad evolucionaria en la medida en que requiere insoslayablemente una semántica realista y una ontología realista. Por un lado, se trata de una semántica realista, porque la verdad es una propiedad de ciertas creencias, mientras que, por otro, se trata de una ontología realista, porque las creencias que tienen la propiedad de ser verdaderas refieren a algo real; siendo así, las creencias verdaderas nos permiten trazar una ontología. ${ }^{11}$

El tercer resultado, aunque parezca ser meramente metodológico, permite apreciar el alcance de la discusión contemporánea sobre teorías de la verdad: la aplicación de la distinción entre consideraciones epistémicas y metafísicas a una teoría de la verdad. Las consideraciones epistémicas sostienen que es evidente $-\mathrm{y}$ hasta ahora éste es el punto que nadie ha negado - que la adquisición de la verdad está sujeta a los constreñimientos epistémicos del agente cognitivo. Sin embargo, las consideraciones metafísicas mantienen intacta la convicción de que la verdad y, con ella, la realidad son independientes de lo que en un momento determinado podamos o queramos pensar o sentir; la metafísica evolucionaria peirceana, al relacionar las nociones de verdad, realidad y evolución, y al desarrollar las ideas de la adecuación de la mente al mundo, de la abducción y del innatismo naturalista, sólo intenta ofrecer buenas hipótesis, desde distintas perspectivas, para mantener la independencia de la verdad y de la realidad respecto de los constreñimientos epistémicos del agente cognitivo, dejando la discusión en un dominio metafísico. Usualmente en la discusión sobre teorías de la verdad ha ocurrido, sin embargo, lo contrario: no se ha distinguido entre consideraciones epistémicas y metafísicas y, por lo mismo, se ha sostenido que una teoría de la verdad es metafísicamente neutral, cuestión que resulta de suyo evidente si se tienen a la vista únicamente consideraciones epistémicas.

Y el cuarto resultado consiste en señalar que la teoría de la verdad evolucionaria es una propuesta que merece ser considerada sobre

\footnotetext{
${ }^{11}$ Sigo la propuesta de Psillos (2005, p. 385), para cuyo desarrollo y discusión se requiere una investigación específica diferente. Sankey $(2008$, p. 18) afirma, en cambio, que el requerimiento semántico es, en realidad, opcional.
} 
todo si se tiene a la vista el trasfondo de la discusión contemporánea sobre el tema; desde Tarski (1944), los teóricos que han abordado el problema se han olvidado en gran medida del carácter eminentemente metafísico del problema de la verdad, vinculado por sí mismo a cuestiones semánticas y ontológicas. Una buena teoría de la verdad no puede desentenderse de esto y caer en la vana satisfacción de ofrecer un informe meramente plausible dentro del contexto de un metalenguaje ficticio. Con esto queda a la vista tal vez lo siguiente: los informes de la verdad predominantes hoy en día tienen en su base una fuerte impronta antirrealista o instrumentalista que, cuando menos, es preciso que sea reconocida en la medida en que subyace en su formulación, arrastrando consecuencias cruciales para la metafísica, la ontología, la epistemología y la semántica, principalmente. Una buena teoría de la verdad no puede desentenderse de su relación acorde o disonante con el resto de los problemas de la filosofía. ${ }^{12}$

\section{BIBLIOGRAFÍA}

Dewey, J., 1911, "The Problem of Truth", The Essential Dewey, vol. 2, Indiana University Press, Bloomington, pp. 101-130.

Fisch, M.H., 1947, "Evolution in American Philosophy", The Philosophical Review, vol. 56, no. 4, pp. 357-373.

Forster, P., 1996, "The Unity of Peirce's Theory of Truth", British Journal of the History of Philosophy, vol. 4, no. 1, pp. 119-147.

George, F.H., 1955, "On a 'Pragmatic' Theory of Truth", The Journal of Philosophy, vol. 52, no. 19, pp. 518-521.

${ }^{12}$ El siguiente trabajo es resultado del Proyecto de Investigación FONDECYT Regular 1095020: Razonamiento basado en modelos (RBM) y generación de hipótesis: un enfoque cognitivo en filosofía de las ciencias a partir de Ronald Giere. Investigador Responsable: profesor Alejandro Ramírez (Departamento de Filosofía, Universidad de Chile); coinvestigadores: profesor Guido Vallejos (Departamento de Filosofía y Centro de Estudios Cognitivos, Universidad de Chile) y profesor Cristian Soto (Departamento de Filosofía, Universidad de Chile).

Agradezco a los profesores Guido Vallejos y Alejandro Ramírez por las observaciones sobre puntos relativos a la teoría de la verdad, la abducción y el realismo en Peirce y en la generación de hipótesis en la filosofía de la ciencia contemporánea. Agradezco también a los expositores asistentes a las IV Jornadas Peirce en Argenti$n a$, realizadas en la Academia Nacional de Ciencias de Buenos Aires, especialmente a la profesora Sara Barrena y al profesor Jaime Nubiola, puesto que de las conversaciones sostenidas y de sus exposiciones extraje diversos vínculos temáticos para continuar la presente investigación. Finalmente, agradezco a los evaluadores anónimos de Crítica: Revista Hispanoamericana de Filosofía por contribuir a mejorar sustancialmente la precisión del argumento. 
Haack, S., 1976, "The Pragmatist Theory of Truth", The British Journal for the Philosophy of Science, vol. 27, no. 3, pp. 231-249.

Hinshaw, Jr., V., 1944, "The Pragmatist Theory of Truth", Philosophy of Science, vol. 11, no. 2, pp. 82?92.

Hookway, C., 2004, "Truth, Reality, and Convergence", en Misak 2004a, pp. 127-149.

- 2002, Truth, Rationality, and Pragmatism. Themes from Peirce, Clarendon Press, Oxford.

Horwich, P., 1996, "Realism and Truth", Noûs, vol. 30, pp. 187-197.

Hynes, C., 2006, "El problema de la unidad de la noción peirceana de verdad", Grupo de Estudios Peirceanos, Universidad de Navarra, España: <http://www.unav.es/gep/IIPeirceArgentinaHynes.html> [última consulta: $24 / 07 / 2010]$.

James, W., 1987, William James: Writings 1902-1910, Library of America, Nueva York.

—_, 1909, "The Meaning of Truth", en James 1987, pp. 821-978.

_ - 1907, "Pragmatism. A New Name for Some Old Ways of Thinking", en James 1987, pp. 479-624.

Kripke, S., 1975, "Outline of a Theory of Truth", The Journal of Philosophy, vol. 72, no. 19, pp. 690-716.

Locke, J., 1979, An Essay Concerning Human Understanding, Oxford University Press, Oxford.

Macintosh, D., 1994, "Partial Convergence and Approximate Truth", The British Journal for the Philosophy of Science, vol. 45, no. 1, pp. 153170.

Misak, C. (comp.), 2004a, The Cambridge Companion to Peirce, Cambridge University Press, Cambridge.

— , 2004b, "C.S. Peirce on Vital Matters", en Misak 2004a, pp. 150174.

- 1991, Truth and the End of Enquiry. A Peircean Account of Truth, Oxford University Press, Oxford.

Peirce, C.S. [W], 1982-2010, Writings of Charles S. Peirce. A Chronological Edition, Indiana University Press, Bloomington, 7 vols.

—_ [EP], 1992-1998, Essential Peirce. Selected Philosophical Writings, vol. 1: 1867-1893, ed. Nathan Houser y Christian Kloesel; vol. 2: 1893-1913, ed. Peirce Edition Project, Indiana University Press, Bloomington.

- [CP], 1934-1958, Collected Papers of Charles S. Peirce, Harvard University Press, Cambridge, Mass.

Psillos, S., 2005, "Scientific Realism and Metaphysics", Ratio (New Series), vol. 18, pp. 385-404.

Quine, W.V.O., 1953, "Two Dogmas of Empiricism”, From a Logical Point of View, Harvard University Press, Cambridge, Mass.

Resnik, D.B., 1992, "Convergent Realism and Approximate Truth", PSA: Proceedings of the Biennial Meeting of the Philosophy of Science Association, vol. 1, pp. 421-434. 
Sankey, H., 2008, Scientific Realism and the Rationality of Science, Ashgate, Aldershot.

Smart, J.J.C., 1995, "A Form of Metaphysical Realism”, The Philosophical Quarterly, vol. 45, no. 180, pp. 301-315. 312.

— 1982, "Metaphysical Realism", Analysis, vol. 42, no. 1, pp. 1-3.

—_, 1963, "Materialism", The Journal of Philosophy, vol. 60, no. 22, pp. 651-662.

Soames, S., 1984, "What Is a Theory of Truth?", The Journal of Philosophy, vol. 81, no. 8, pp. 411-429.

Soto, C., 2009, "Pragmatismo y realismo científico en Peirce", Cuadernos de Filosofía, Universidad de Concepción (Chile), no. 27, pp. 23-45.

Tarski, Alfred, 1944, "The Semantic Conception of Truth and the Foundations of Semantics", Philosophy and Phenomenological Research, vol. 4, pp. 341-375.

Törnudd, A., 1915, “Types of Pragmatist Theory of Truth", The Journal of Philosophy, Psychology and Scientific Methods, vol. 12, no. 18, pp. 491500.

Ullian, J.S., 1991, “Truth”, Journal of Aesthetic Education, vol. 25, no. 1, pp. $57-65$.

Vallejos, G., 1996, Peirce: pragmatismo, semiótica y realismo, Publicaciones Especiales del Departamento de Filosofía, Universidad de Chile, Santiago de Chile, no. 59; también disponible en: <http://www.unav.es/ gep/ArticulosOnLineEspanol.html> [última consulta: 24/07/2010], y en: $<$ http://www.antroposmoderno.com/antro-articulo.php?id_articulo=589> [última consulta: 24/07/2010].

Wiener, P., 1946, "The Evolutionism and the Pragmaticism of Peirce", The Journal of the History of Ideas, vol. 7, no. 3, pp. 321-350.

Wiggins, D., 2004, "Reflections on Inquiry and Truth Arising from Peirce's Method for the Fixation of Belief", en Misak 2004a, pp. 87-126.

Recibido el 11 de enero de 2010; revisado el 26 el julio de 2010; aceptado el 18 de agosto de 2010. 